\title{
The Armc10/SVH gene: genome context, regulation of mitochondrial dynamics and protection against $A \beta$-induced mitochondrial fragmentation
}

\author{
R Serrat ${ }^{1,2,7}$, S Mirra ${ }^{1,2,7}$, J Figueiro-Silva ${ }^{2,3}$, E Navas-Pérez ${ }^{4}$, M Quevedo ${ }^{1,2}$, G López-Doménech ${ }^{1,2}$, P Podlesniy ${ }^{2,3}, F_{\text {Ulloa }}{ }^{1,2}$, \\ J Garcia-Fernàndez ${ }^{4}$, Trullas $^{2,3}$ and E Soriano ${ }^{\star, 1,2,5,6}$
}

Mitochondrial function and dynamics are essential for neurotransmission, neural function and neuronal viability. Recently, we showed that the eutherian-specific Armcx gene cluster (Armcx1-6 genes), located in the X chromosome, encodes for a new family of proteins that localise to mitochondria, regulating mitochondrial trafficking. The Armcx gene cluster evolved by retrotransposition of the Armc10 gene mRNA, which is present in all vertebrates and is considered to be the ancestor gene. Here we investigate the genomic organisation, mitochondrial functions and putative neuroprotective role of the Armc10 ancestor gene. The genomic context of the Armc10 locus shows considerable syntenic conservation among vertebrates, and sequence comparisons and CHIP-data suggest the presence of at least three conserved enhancers. We also show that the Armc10 protein localises to mitochondria and that it is highly expressed in the brain. Furthermore, we show that Armc10 levels regulate mitochondrial trafficking in neurons, but not mitochondrial aggregation, by controlling the number of moving mitochondria. We further demonstrate that the Armc10 protein interacts with the KIF5/Miro1-2/Trak2 trafficking complex. Finally, we show that overexpression of Armc10 in neurons prevents $A \beta$-induced mitochondrial fission and neuronal death. Our data suggest both conserved and differential roles of the Armc10/Armcx gene family in regulating mitochondrial dynamics in neurons, and underscore a protective effect of the Armc10 gene against A $\beta$-induced toxicity. Overall, our findings support a further degree of regulation of mitochondrial dynamics in the brain of more evolved mammals.

Cell Death and Disease (2014) 5, e1163; doi:10.1038/cddis.2014.121; published online 10 April 2014

Subject Category: Neuroscience

Mitochondria are essential organelles for many biological processes, including respiration, energy production and cell viability. Owing to the length of neuronal processes (axons and dendrites) and the function of mitochondria in neurotransmission, calcium buffering and neural integration, the correct trafficking and distribution of these organelles is crucial for neuronal function. ${ }^{1,2}$ Mitochondrial trafficking in neurons is mediated by kinesin and dynein motors and by several GTPases and adaptor proteins, including Miro1-2, Trak2 and mitofusins. ${ }^{3-7}$ In fact, impaired distribution and function of mitochondria and mutations in mitochondrialrelated motors and proteins have been found to be implicated in numerous neurodegenerative diseases, including Parkinson, Alzheimer and Huntington diseases, as well as in rare disorders such as Charcot Marie-Tooth disease..$^{2,8-10}$

In a previous study, we provided evidence that proteins encoded by the eutherian-specific Armcx gene cluster in the $\mathrm{X}$ chromosome (Armcx1-6, Armadillo repeat containing
X-linked) localise to mitochondria. ${ }^{11}$ Furthermore, our study demonstrated that at least one member of this family, Armcx3, regulates mitochondrial dynamics in neurons and interacts with the kinesin/Miro/Trak2 protein complex responsible for mitochondrial trafficking. ${ }^{12-14}$ Interestingly, this interaction is $\mathrm{Ca}^{2+}$-dependent and Armcx3 was found to control mitochondrial aggregation, dynamics and trafficking in neurons. ${ }^{11}$ As the Armcx gene cluster is highly expressed in the brain and is specific to eutherian mammals, the findings of the above study indicated a further degree of molecular complexity and regulation of mitochondrial dynamics events, specifically in the brains of eutherians - the most evolved mammals.

The Armcx gene cluster evolved by retrotransposition of an Armc10 mRNA. Thus, the Armc10/SVH gene is present in a single copy in all the vertebrate genomes analysed, including eutherian mammals, and it represents the closest phylogenetic relative to the Armcx gene cluster. Phylogenetic analyses have identified the Armc10 gene as being the sister

\footnotetext{
${ }^{1}$ Department of Cell Biology, University of Barcelona, Barcelona E-08028, Spain; ${ }^{2}$ Centro de Investigación Biomédica en Red sobre Enfermedades Neurodegenerativas (CIBERNED, ISCIII), Barcelona E-08028, Spain; ${ }^{3}$ Neurobiology Unit, Institut d'Investigacions Biomèdiques de Barcelona, CSIC, IDIBAPS, Barcelona E-08036, Spain; ${ }^{4}$ Department of Genetics, University of Barcelona, Barcelona E-08028, Spain; ${ }^{5}$ Fundación CIEN, Vallecas E-28031, Madrid, Spain and ${ }^{6}$ Vall d'Hebron Institut de Recerca, Barcelona E-08035, Spain

${ }^{*}$ Corresponding author: E Soriano, Department of Cell Biology, University of Barcelona, Barcelona E-08028, Spain. Tel: +34 93 4037117; Fax: + 34 93 4037114; E-mail: esoriano@ub.edu

${ }^{7}$ These authors contributed equally to this work.

Keywords: mitochondrial dynamics; $A \beta$-toxicity; mitochondrial fission; mitophagy

Abbreviations: $\mathrm{A} \beta$, amyloid-beta; Armcx, armadillo repeat containing X-linked; ChIP, chromatin immunoprecipitation; DUF, domain of unknown function; GFP, green fluorescent protein; KHC, kinesin heavy chain; KIF, kinesin superfamily protein; SVH, splicing variant involved in hepatocarcinogenesis; WB, western blot

Received 05.7.13; revised 10.2.14; accepted 24.2.14; Edited by A Verkhratsky
} 
group to all Armcx genes. ${ }^{11}$ Here, we show that the Armc10 locus resides in a large syntenic region conserved among vertebrates and contains at least three conserved enhancers; we also demonstrate that the Armc10/SVH protein is highly expressed in developing and adult neurons, where it localises to nuclei and mitochondria. Furthermore, we show that the Armc10/SVH protein regulates mitochondrial trafficking, interacting with the kinesin/Miro/Trak2 complex, and that overexpression of Armc10 prevents $\mathrm{A} \beta$-induced mitochondrial fragmentation. Our data indicate a conserved role of the Armc10/Armcx gene family in the regulation of mitochondrial dynamics and transport in neurons and suggest a protective role of the Armc10/SVH protein against $\mathrm{A} \beta$-induced toxicity.

\section{Results}

Genome context and regulation conservation in the Armc10 locus. To analyse conservation and changes in the genomic context of the Armc10 locus in vertebrates, we studied the synteny of the surrounding genes in selected species, looking for the conservation of five genes upstream and downstream of Armc10 (Figure 1). Synteny was found to be well conserved in all tetrapods analysed. The only exception was found in the human genome, which did not show conservation of the two most upstream genes. Following the teleost-specific genome duplication event, zebrafish only possesses single cognates of Armc10 and surrounding genes, resulting in partial conservation of synteny, scrambled in chromosomes 4 and 25.

Next, the conservation of non-coding regions in the genomic context of Armc10 was analysed using VISTA, and the results were compared with available information on the histone code and transcription factor ChIP-Seq from the ENCODE Project (Figure 2). Taking the human genome as reference, no sequence conservation was found beyond mammals. However, a significant peak with enhancer histone marks was observed in one of the introns of the bystander gene FBXL13, as well as two weak peaks conserved in both placental mammals and marsupials, one located in an intron of Armc10 and another in an intron of the NAPEPLD gene (Figure 2). Conserved regions with the enhancer histone mark, H3K4me1, but without a signal for the promoter histone mark, $\mathrm{H} 3 \mathrm{~K} 4 \mathrm{me} 3$, may be considered as putative enhancers. ${ }^{15}$

The genome context of the Armc10 gene is clearly in contrast to the $X$ chromosome Armcx $1-6$ cluster, ${ }^{11}$ which originated after a retrotransposition event at the base of the eutherian tree; hence, the Armcx cluster does not show any synteny relationship, nor does it share any regulatory sequences, with the Armc10 genome context. Furthermore, the transcription units are evidently unrelated: the mouse Armc10 protein sequence is encoded by seven exons localised in chromosome $7 q 11.22,{ }^{16}$ whereas the Armcx proteins are all encoded in a single exon.

Neuronal expression and mitochondrial localisation of the Armc10/SVH protein. The available RNA-seq data show that the Armc10 gene is expressed in several tissues - but predominantly in the brain - in zebrafish, Xenopus, chicken, mouse and humans (Figure 3a). However, these data do not take alternative spliced isoforms into account, and it has been proposed that up to six isoforms arise from alternative splicing of the Armc10 gene (Figure 3b). Of these, four have been detected in human hepatocarcinoma cells (Armc10 A-D). ${ }^{16}$ The Armc10 protein contains a transmembrane domain at the N-terminus (aa 7-29), a putative cleavage site (aa 30-36) and a flanking basic region near the transmembrane region (similar to that found in Tom20 and $\mathrm{Bcl}-\mathrm{w})$, which predicts putative targeting to the outer mitochondrial membrane. ${ }^{17}$ Full-length Armc10 (A isoform) contains up to six armadillo domains arranged in a DUF634 domain (aa 85-337), which are partially deleted in some isoforms. The protein also contains nuclear export signals and several potential phosphorylation sites (Figure 3b).

Western blot (WB) experiments revealed that an anti-Armc10 antibody recognised up to four bands in untransfected HEK293T cells (Figure 3c), which presumably correspond to different Armc10 isoforms. WBs of embryonic and adult brain lysates showed a prominent $31-\mathrm{kDa}$ band,

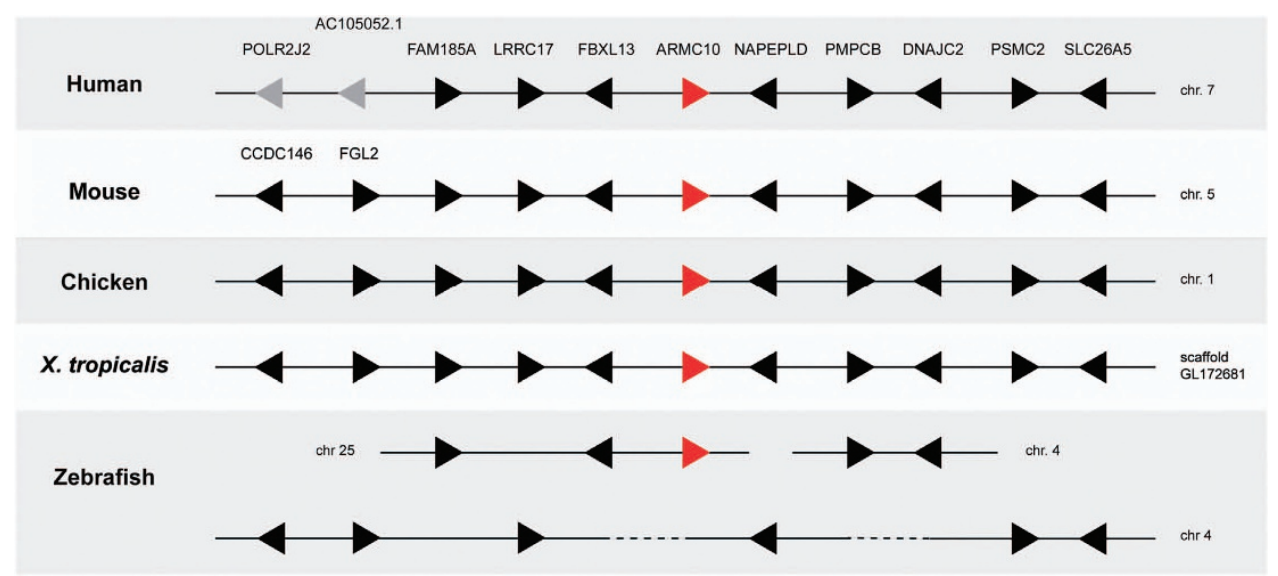

Figure 1 Conserved gene synteny surrounding the Armc10 locus in vertebrates. Extended synteny of Armc10 is conserved in all tetrapods with the exception of humans. In zebrafish, the synteny is lost, most probably due to the fish-specific genome duplication event and subsequent specific gene losses. Arrows indicate genes (red: Armc10, black: syntenic genes, grey: non-syntenic genes) and direction of transcription. Dashed lines indicate large genomic regions 


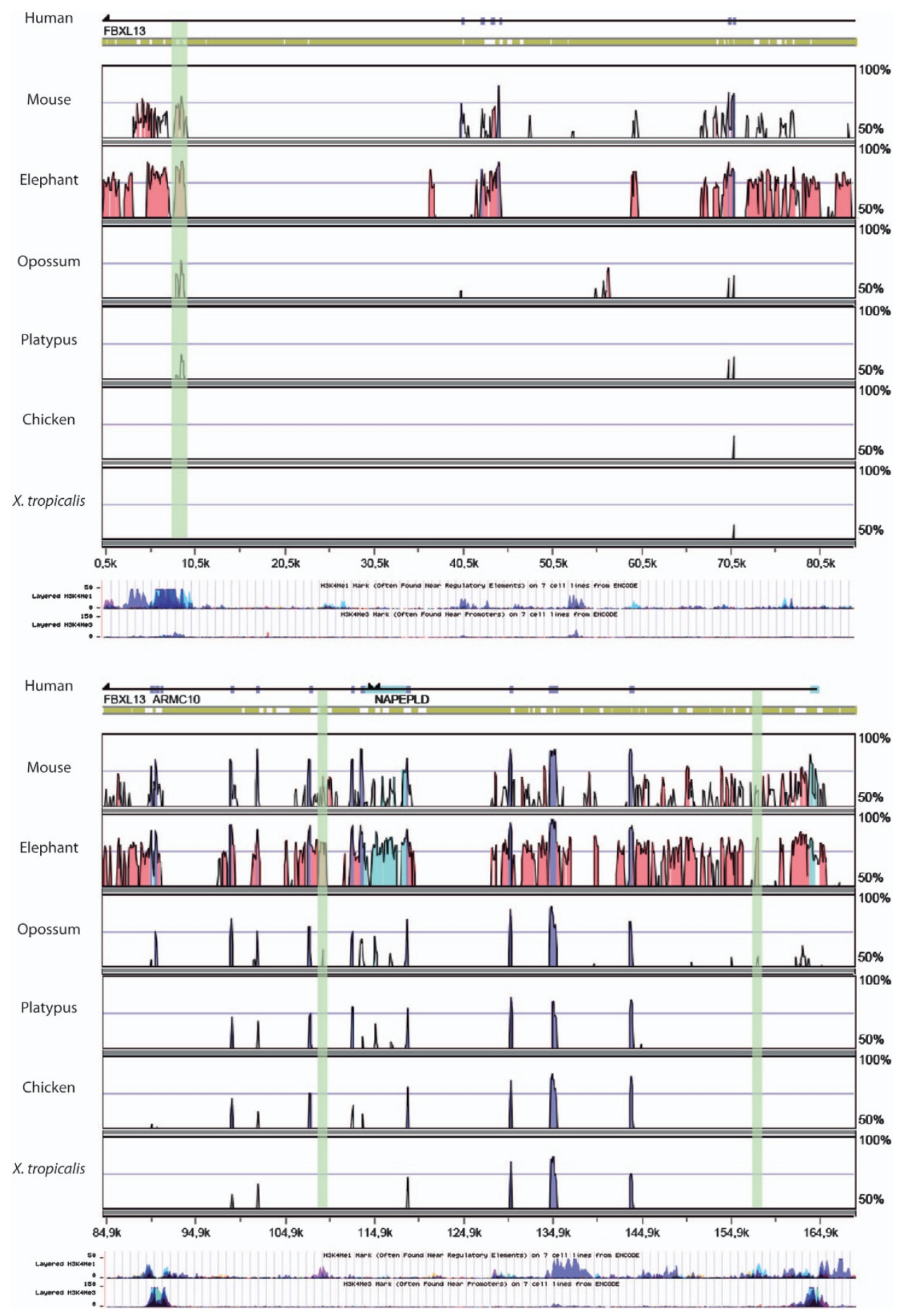

Figure 2 VISTA plots of the alignment of the region close to the Armc10 locus among six tetrapod species. The genomic region analysed extends $165 \mathrm{~kb}$ (first half in upper panel, second half in lower panel). H3K4me3 and H3K4me1 histone marks from the ENCODE Project on seven cell lines for the region in human are shown below the VISTA plot. Peaks (blue: exons, light blue: UTR, pink: non-coding region) indicate regions with a conservation of at least $70 \%$ and a minimum width of $100 \mathrm{bp}$. Non-coding peaks with enhancer histone marks are highlighted in pale green

which probably corresponds to the Armc10 C and/or E isoforms. We analysed the regional and cellular patterns of protein distribution using immunohistochemistry. Thus, the Armc10 protein showed wide expression both at developmental (E16-P5) and adult (Figure 3d-h) stages, labelling many neuronal populations. Immunostaining was prominent in the cerebral cortex, hippocampus, thalamus and cerebellum. In most developing and adult neurons, Armc10 signals were prominent in neuronal nuclei and fainter staining was detected in neuronal perikarya and in the neuropil. 
4

a

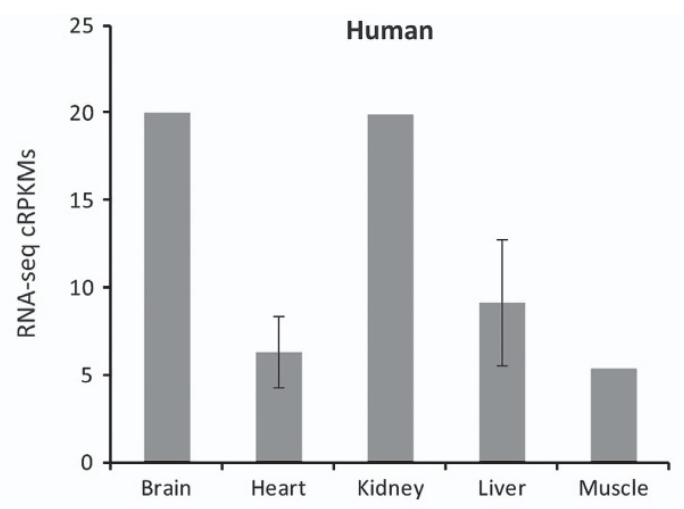

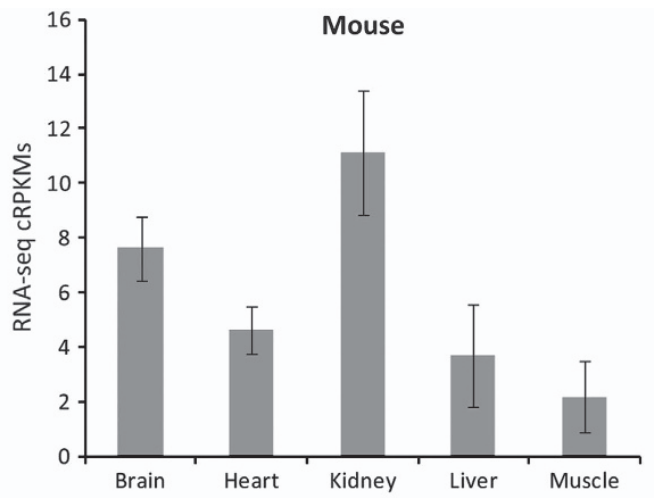

b

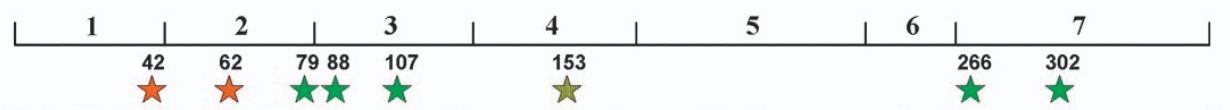
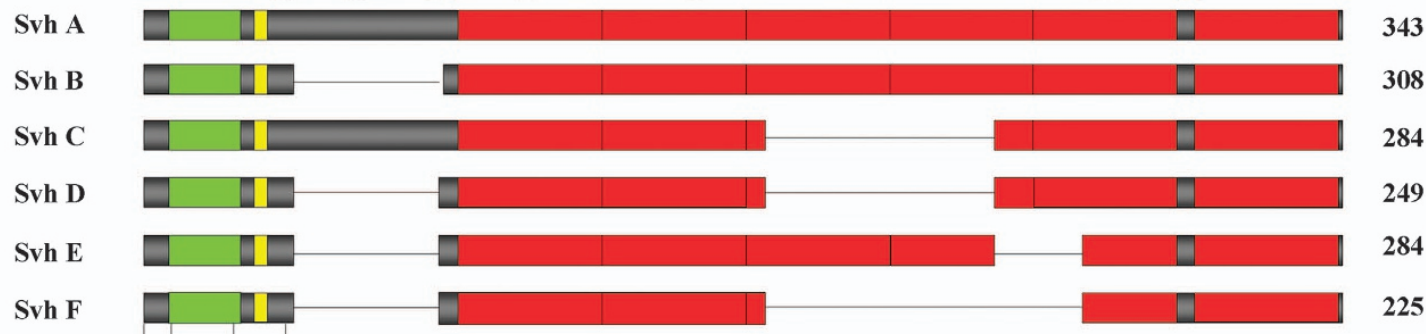

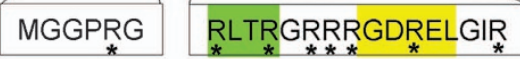

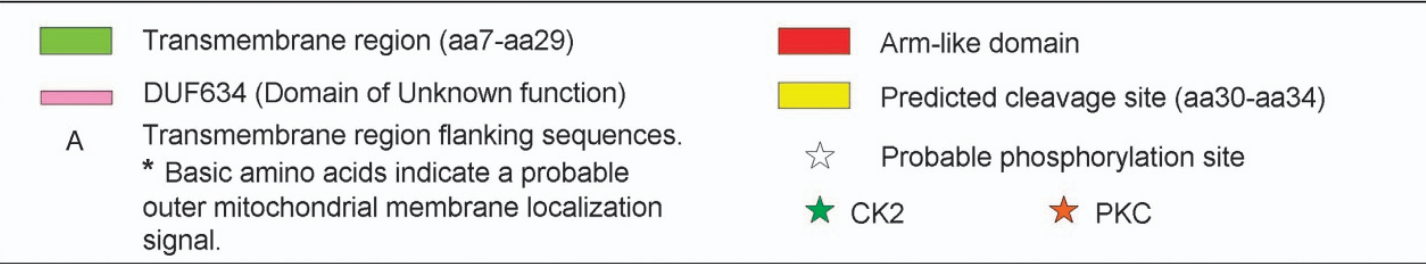

c
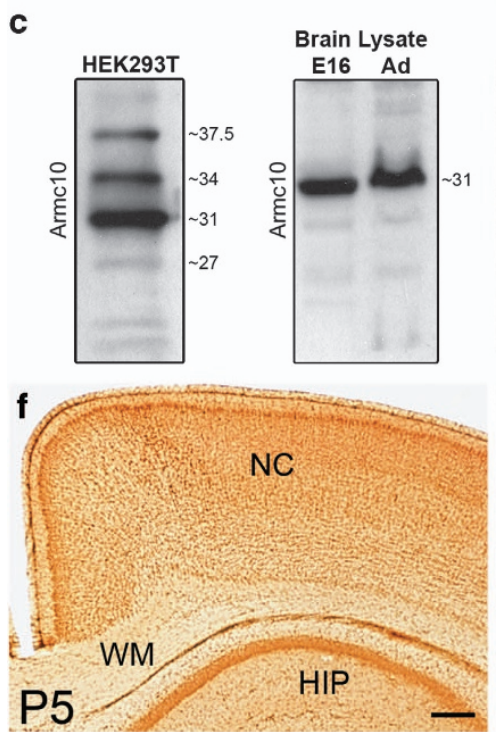

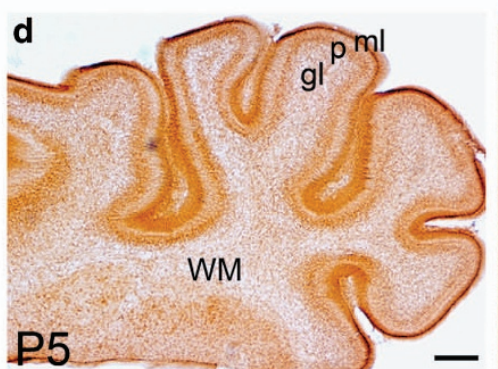

D5 $\quad-A d$

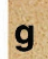

g

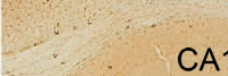

CA1

CA3

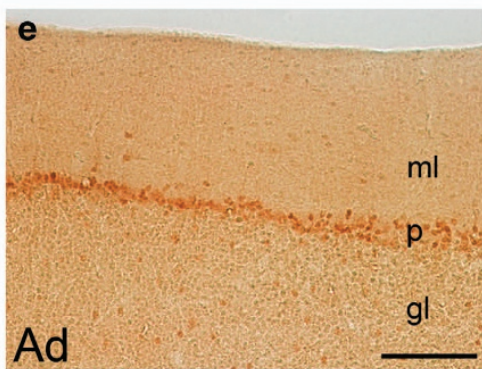

h

II-III

IV

Ad 
To gain a better understanding of the subcellular distribution of endogenous Armc10 protein, we immunostained HEK293T cells and hippocampal primary cultures. These cells exhibited marked co-staining of the Armc10 protein with mitochondrial markers in both cell bodies and neurites (Figure $4 a-c$ ). To confirm this localisation, the protein levels of Armc10 were examined by WB after brain mitochondrial isolation; these experiments showed a marked mitochondrial enrichment of the Armc10 protein (Supplementary Figure 1). The above data indicate that, as with the Armcx3 protein, Armc10 is widely expressed in the nervous system, where it shows a mainly bimodal localisation in mitochondria and the cell nucleus.

\section{Armc10 protein regulates mitochondrial aggregation} and trafficking. To examine the role of the Armc10 protein in mitochondrial dynamics, we transfected HEK293T cells and hippocampal neurons with a GFP-tagged Armc10 cDNA. In agreement with the above results, transfection of Armc10 yielded a preferential localisation of this protein in mitochondria (Figure 4d and e). Armcx3 overexpression has been found to lead to mitochondrial aggregation. ${ }^{11}$ To study whether this also held true for the Armc10 protein, we analysed the mitochondrial phenotypes induced by Armc10-GFP overexpression. In control HEK293T cells, most transfected cells (MitDsRed) displayed a regularly arranged mitochondrial meshwork (as in Figure $4 \mathrm{a}$ and f). In contrast, up to $78 \%$ of cells overexpressing Armc10 displayed abnormal mitochondrial phenotypes, whereas $29 \%$ of cells showed strong phenotypes in which mitochondria were aggregated in a single cluster near the cell nucleus (Figure $4 \mathrm{e}$ and f) and $49 \%$ of transfected cells displayed milder degrees of mitochondrial aggregation involving several clusters in which individual mitochondria were still visible (Figure $4 d$ and f).

However, overexpression of Armc10-GFP in cultured hippocampal neurons only caused mild mitochondrial aggregation, without leading to strongly aggregating phenotypes. Thus, only $33 \%$ of transfected neurons displayed mildly aggregating phenotypes, with small clusters of mitochondria present in both the cell body and neurites (Figure 4g-i). The differential aggregation of phenotypes caused by Armc10 overexpression in HEK293T cells and neurons supports the notion of a differential regulation of mitochondrial dynamics and aggregation in these two cell types.

To determine whether Armc10 protein levels alter mitochondrial trafficking in neurons, we first transfected hippocampal neurons with Armc10-GFP cDNA or with the control vector MitDsRed. Two days later, axons from transfected neurons were identified and recorded for $15 \mathrm{~min}$, and mitochondrial dynamics were quantified using kymographs. ${ }^{11,12}$ Control neurons displayed active mitochondrial trafficking ( $\sim 30 \%$ of mitochondria) in anterograde and retrograde directions (Figure $5 \mathrm{a}$ and b). Individual mitochondria moved at an average velocity of $\sim 0.16-0.19 \mu \mathrm{m} / \mathrm{s}$ (for retrograde and anterograde transport, respectively; Figure 5b). Overexpression of Armc10-GFP resulted in a marked decrease in the percentages of motile mitochondria in both anterograde and retrograde directions. In contrast, the velocity of the mitochondria that remained motile was unaffected by Armc10 overexpression (Figure 5b). These results were independent of mitochondrial numbers in axons (Supplementary Figure 2). To study the contribution of endogenous Armc10 protein to mitochondrial transport, we designed shRNA sequences corresponding to the C-terminal region of Armc10 in order to knockdown all endogenous Armc10 isoforms (Figure 5c; see also Figure $3 b$ ). In addition, Armc10 downregulation resulted in a percentage of motile mitochondria that was reduced by more than half in the retrograde and anterograde directions of transport, in comparison with control neurons transfected with a scrambled sequence. Interestingly, the velocity of the mitochondria that remained motile was unaffected by the levels of Armc10 protein expression (Figure $5 d$ and e). These findings indicate that Armc10 protein levels regulate mitochondrial trafficking in neurons mainly by controlling the percentage of motile mitochondria.

\begin{abstract}
Armc10 protein interacts with the $\mathrm{KHC} / \mathrm{Miro} / \mathrm{Trak2}$ complex. Mitochondrial trafficking in neurons is believed to be controlled by the kinesin 1 motor protein and by the adaptor and Rho GTPase proteins Trak2 and Miro1-2, respectively. ${ }^{5-7}$ We thus studied whether the Armc10 protein interacts with the kinesin/Miro/Trak2 trafficking complex. Immunofluorescence analyses in transfected HEK293T cells showed strong colocalisation of Armc10 with Miro2 and Trak2, and partial overlapping with KIF5C (Figure 6a and Supplementary Figure 3). Co-immunoprecipitation experiments in HEK293T cells transfected with Armc10-GFP and either Miro1-myc or Miro2-myc cDNAs revealed that Miro proteins were detected by WB in lysates immunoprecipitated with anti-GFP antibodies. Conversely, immunoprecipitation with anti-myc antibodies revealed the Armc10-GFP protein. No co-immunoprecipitation was detected when cells were transfected with either Miro1-2-myc or Armc10-GFP DNA alone (Figure $6 \mathrm{~b}$ and not shown). Co-immunoprecipitation experiments with the mitochondrial adaptor protein Trak2 also revealed co-association with the Armc10 protein (Figure 6b). In contrast, we did not detect co-immunoprecipitation of the Armc10-GFP protein with KIF5C, KIF5A or KIF5B in transfected HEK293T cells (Figure 6b and not shown).
\end{abstract}

Figure 3 Schematic representation of Armc10 isoforms and expression of the Armc10 protein. (a) RNA-seq data showing the expression of Armc10 transcripts in different adult human and mouse tissues. (b) Structure, domains and putative phosphorylation sites of six Armc10 (A-E) isoforms. They all have a potential N-terminal transmembrane region and a predicted cleavage site. The C-terminal domain (DUF634) consists of up to six Arm-like tandem repeats, the distribution of which depends on the alternative splicing pattern of the Armc10 gene (see numbers above representing exons). Stars point to putative phosphorylation sites by CK2 and PKC kinases. (c) WB showing that an anti-Armc10 antibody recognises four Armc10 bands, presumably corresponding to isoforms (37.5, 34, 31 and $27 \mathrm{kDa}$ ) in HEK293T cells and one single band, corresponding to isoforms of $31 \mathrm{kDa}$, in brain lysates from E16 and adult mice. (d-h) Coronal sections of P5 and adult mouse brain showing expression pattern of the Armc10 protein in the cerebellum ( $\mathbf{d}$ and $\mathbf{e})$, neocortex ( $\mathbf{f}$ and $\mathbf{h}$ ) and the hippocampus $(\mathbf{g})$. Boxed area in $(\mathbf{h})$ shows nuclear localisation of the Armc10 protein in the neocortex. Ad: Adult; CA1-CA3: hippocampal regions CA1-CA3; DG: dentate gyrus; gl: granular layer; HIP: hippocampus; II-III-IV: neocortical layers; ml: molecular layer; NC: neocortex; p: purkinje cell layer; P5: post-natal day 5; WM: white matter. Scale bars: $100 \mu \mathrm{m}(\mathbf{d}-\mathbf{h}) ; 50 \mu \mathrm{m}$ (boxed area in h) 

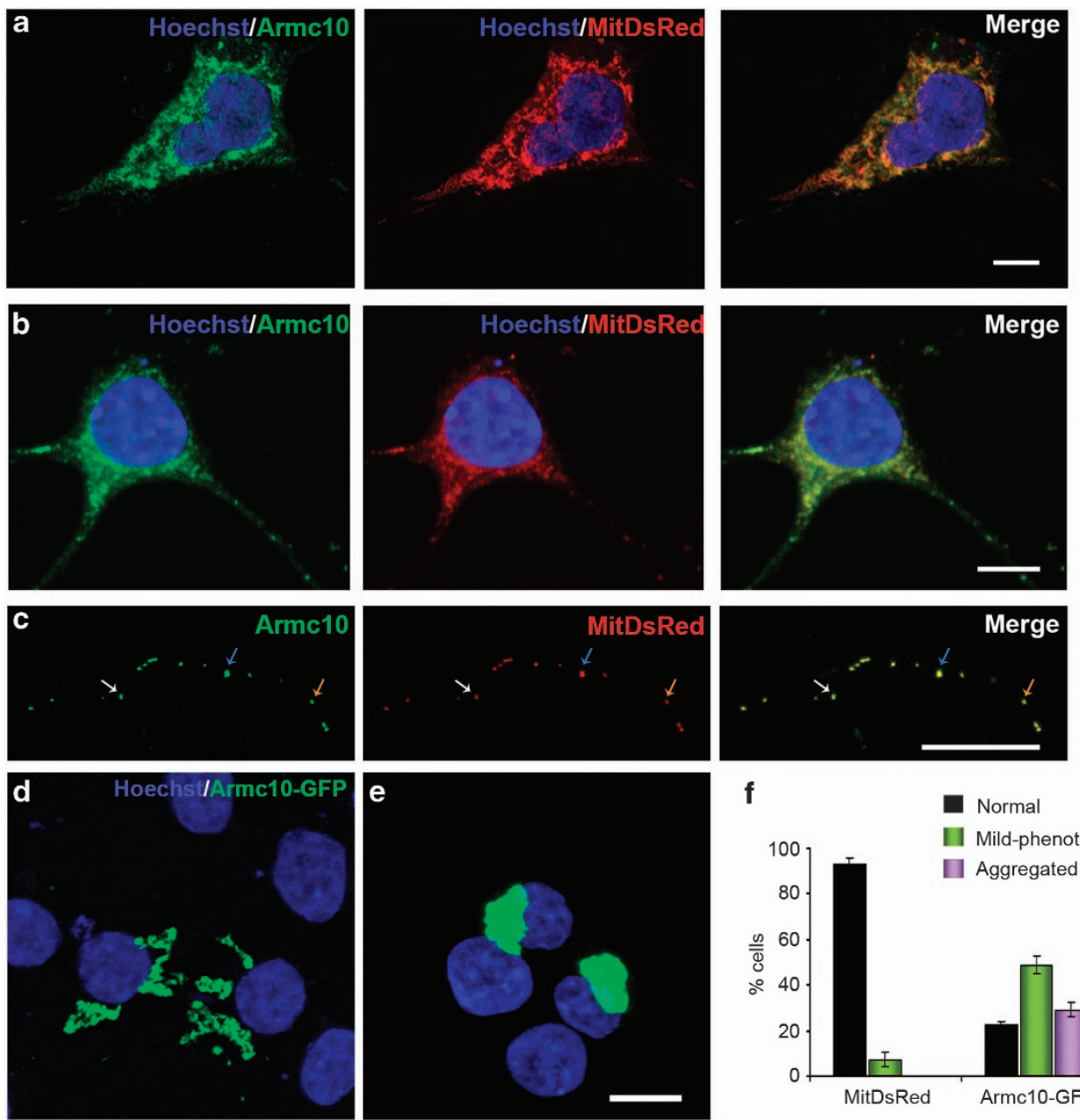

$\mathbf{f}$
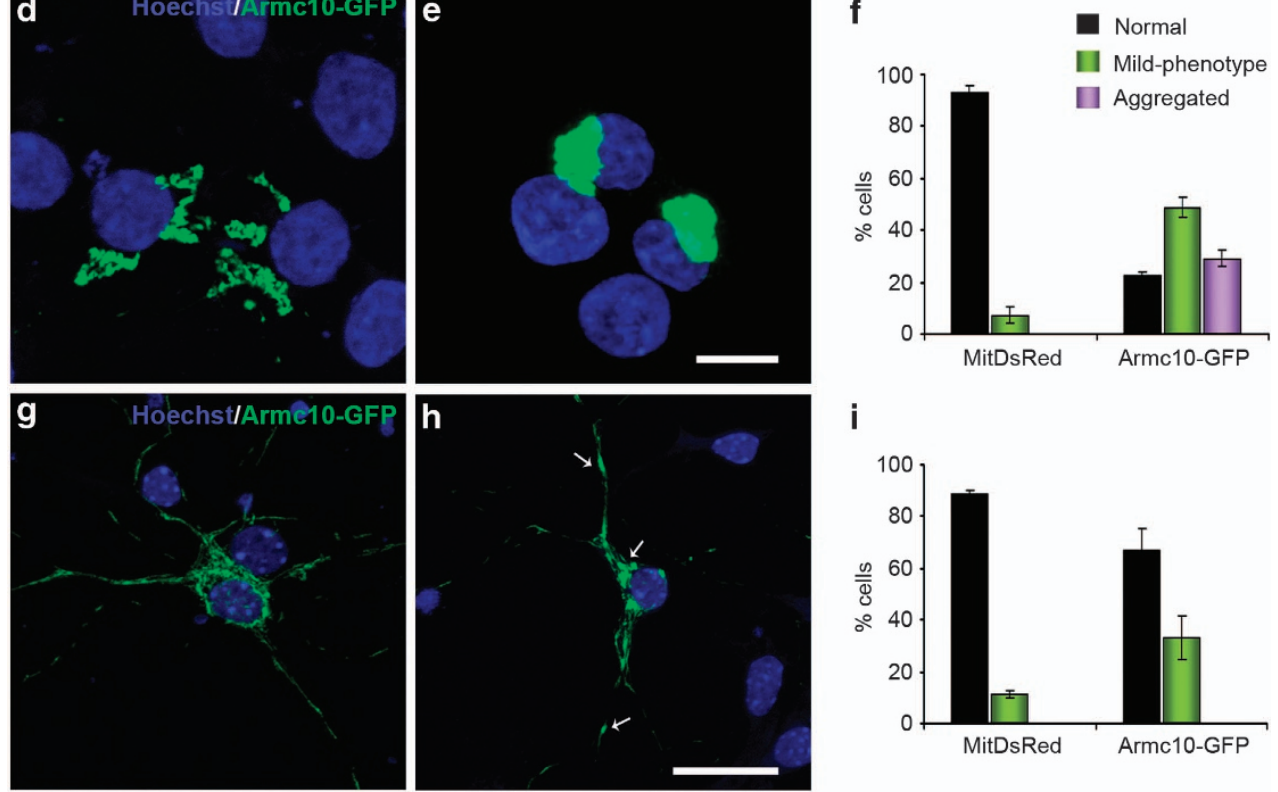

Figure 4 Armc10 colocalises with mitochondria and its overexpression alters mitochondrial distribution, thus leading to perinuclear aggregation in HEK293T cells but not in neurons. (a-c) Confocal images showing mitochondrial colocalisation of endogenous Armc10 (green) with the mitochondrial marker MitDsRed (red) in HEK293T cells (a) and in six DIV hippocampal neurons (b, axonal projection in $\mathbf{c}$ ). (d-f) Overexpression of Armc10-GFP in HEK293T cells induces alterations in the distribution of mitochondria when compared with the expression of MitDsRed control vector. These phenotypes were classified as 'normal', 'mild' and 'aggregated'. Examples of HEK293T cells displaying mildly (d) and strongly (e) aggregating mitochondrial phenotypes. (f) Histogram illustrating percentages of HEK293T mitochondrial phenotypes after transfection with MitDsRed or Armc10-GFP. (g-i) Hippocampal neurons overexpressing an Armc10-GFP cDNA display normal mitochondrial phenotype (g) or a mildly aggregated phenotype (h) with small clusters of mitochondria in cell bodies and neurites (arrows). (i) Histogram illustrating percentages of hippocampal neurons showing normal and mild phenotypes after transfection with MitDsRed or Armc10-GFP. We counted 62-157 HEK293T cells and 35-41 neurons per condition. Data represent mean \pm S.E.M. of three independent experiments. Nuclei were stained with bisbenzimide (blue). Scale bars: $10 \mu \mathrm{m}$ (a-d); $20 \mu \mathrm{m} \mathrm{(b,} \mathrm{c,} \mathrm{f} \mathrm{and} \mathbf{g}$ )

To support these findings, we performed co-immunoprecipitation assays with adult brain lysates (Figure 6c). The Armc10 protein was found to interact both with Miro1 and Trak2, but not with KHC, the heavy chain of kinesin 1. Reverse immunoprecipitation, with Trak2 and Miro1 antibodies, also yielded the Armc10 protein (Figure 6c).
Overexpression of Armc10 protects against A $\beta$-induced neuronal death and mitochondrial fragmentation. Dysfunction of mitochondrial dynamics is commonly associated with neural diseases. For instance, $A \beta$ has been reported to induce mitochondrial fragmentation and fission, which is believed to contribute to neural 
a

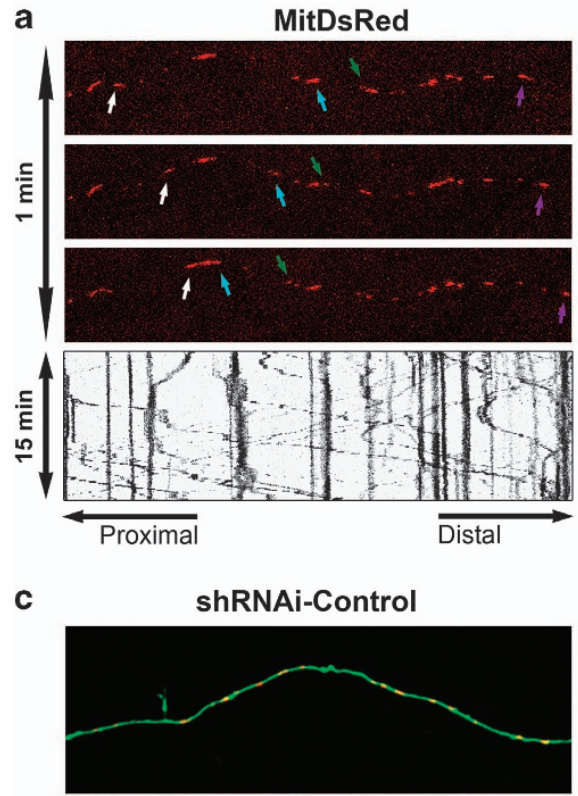

d

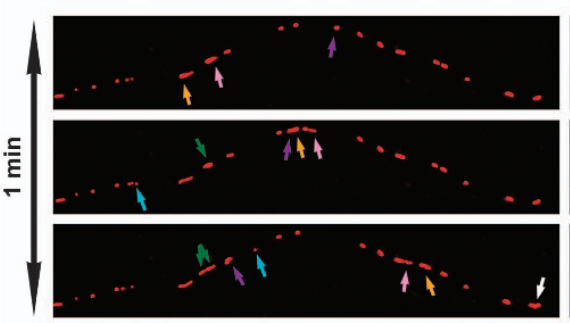

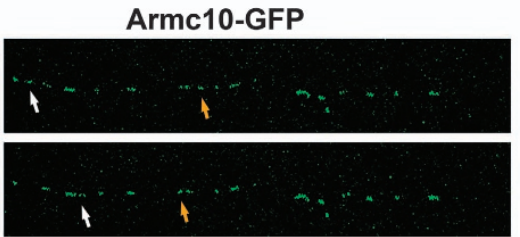
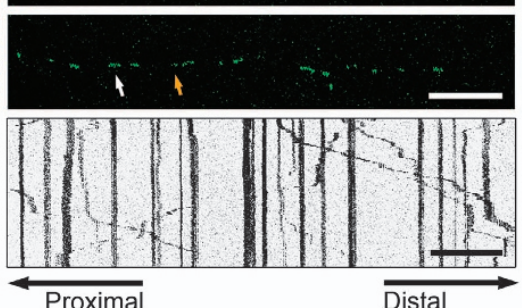

Proximal
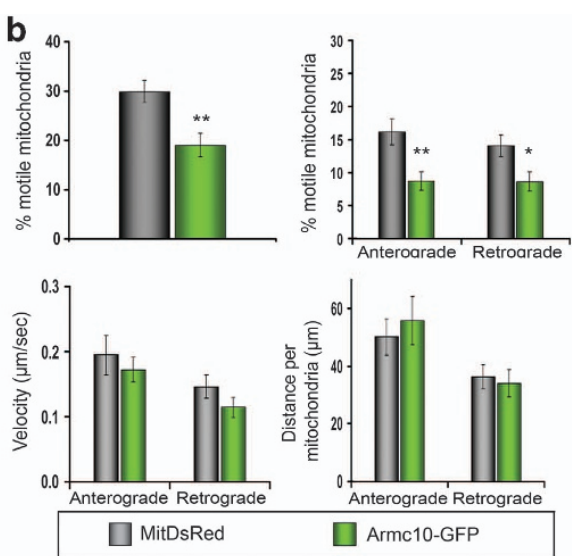
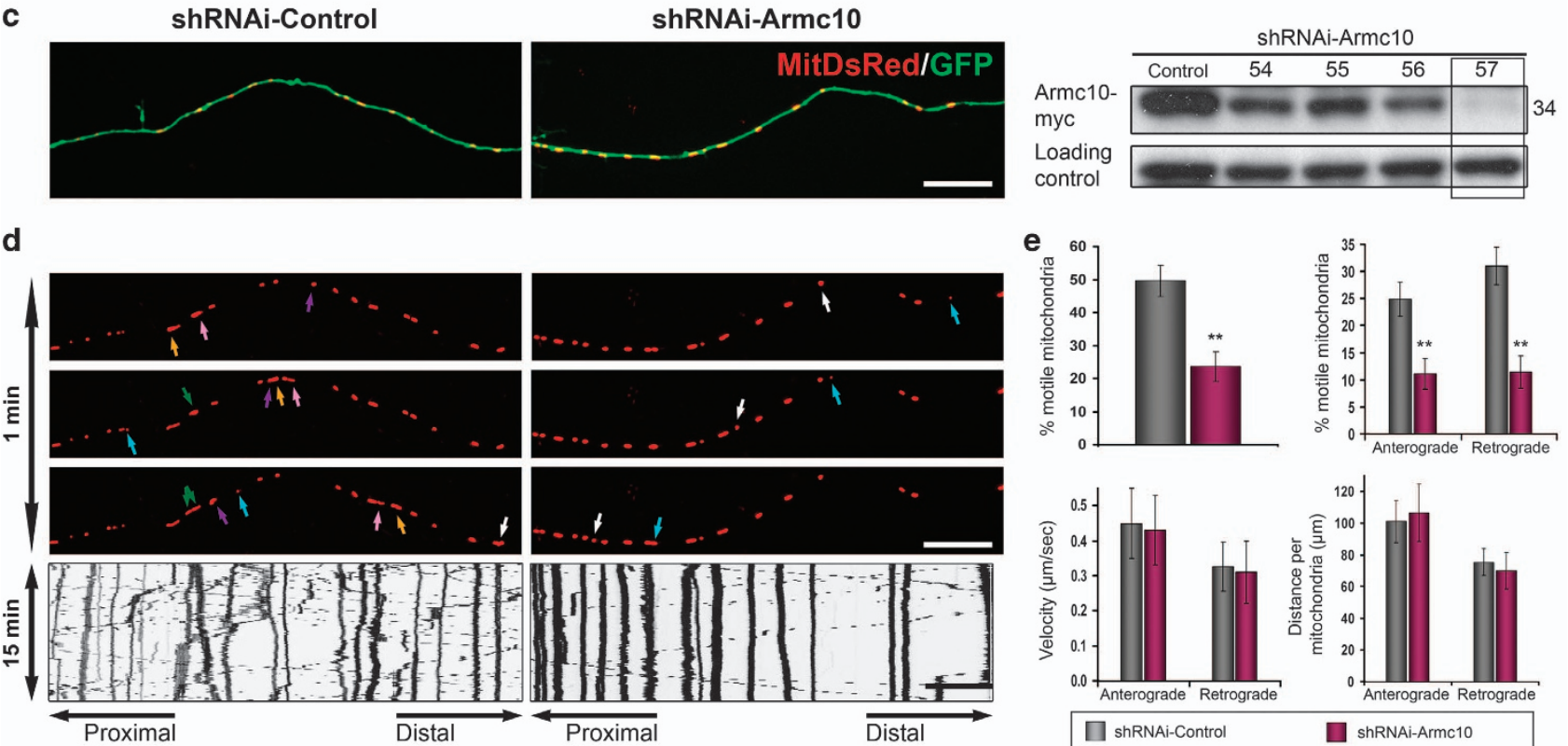

Figure 5 Armc10 protein levels regulate mitochondrial axonal transport in hippocampal neurons. (a) Series of three representative confocal images, taken every $30 \mathrm{~s}$, of live axons overexpressing MitDsRed or Armc10-GFP fusion protein. Representative kymographs giving the full-time acquisition periods are shown in lower panels. (b) Graphical representation of the percentages of motile mitochondria, and the velocity and distance covered by individual mitochondria measured in kymographs for control and overexpressing neurons. Data represent the mean \pm S.E.M. (c) Examples of axons co-expressing MitDsRed plus an Armc10-specific shRNAi or a control shRNAi. In the right panel, HEK293T cells were co-transfected with an Armc10-myc expression construct and pLVTHM, containing several Armc10-specific shRNAi sequences (54-57) and a scrambled control; cells were lysated and processed for WB with an anti-myc antibody. One Armc10-specific shRNAi sequence (57) downregulated Armc10 protein expression and was selected to perform the silencing experiments. (d) Series of representative confocal images, taken every $30 \mathrm{~s}$, of live axons overexpressing MitDsRed together with an Armc10-specific shRNAi or a control shRNAi. Representative kymographs giving the full-time acquisition periods are shown in lower panels. (e) Graphical representation of the percentages of motile mitochondria, and the velocity and distance covered by individual mitochondria measured in kymographs for shRNAi-control and shRNAi-Armc10-overexpressing neurons. Data represent the mean \pm S.E.M. Scale bars: $10 \mu \mathrm{m}$

dysfunction and neuronal death. ${ }^{18}$ To investigate whether the Armc10 protein influences this process, we treated cultured neurons overexpressing Armc10 with $\mathrm{A} \beta(2 \mu \mathrm{M})$ and determined mitochondrial sizes. As already described elsewhere, ${ }^{19}$ incubation with $\mathrm{A} \beta$ induced mitochondrial fragmentation in hippocampal neurons, compared with vehicle-treated neurons (Figure $7 a, b$ and e). Armc10overexpressing neurons displayed slightly larger mitochondria than control neurons. Moreover, overexpression of Armc10 in hippocampal neurons completely abolished $\mathrm{A} \beta$-induced mitochondrial fragmentation (Figure $7 \mathrm{~d}$ and $\mathrm{e}$ ).

To further confirm the protective effect of Armc10 against $\mathrm{A} \beta$ toxicity, we also evaluated the percentage of cell death in hippocampal cultures. The overexpression of Armc10 caused a significant reduction $(>66 \%)$ in the number of dead cells induced after $\mathrm{A} \beta$ treatment $(2 \mu \mathrm{M})$, in comparison with control neurons (Figure 7f). Together, these results indicate that Armc10 protein levels not only modulate mitochondrial dynamics under control conditions but also protect neurons and mitochondria from insult-induced toxicity, fragmentation and cell damage.

\section{Discussion}

The eutherian-specific Armcx gene cluster arose during evolution by retrotransposition of an Armc10 mRNA, a gene 

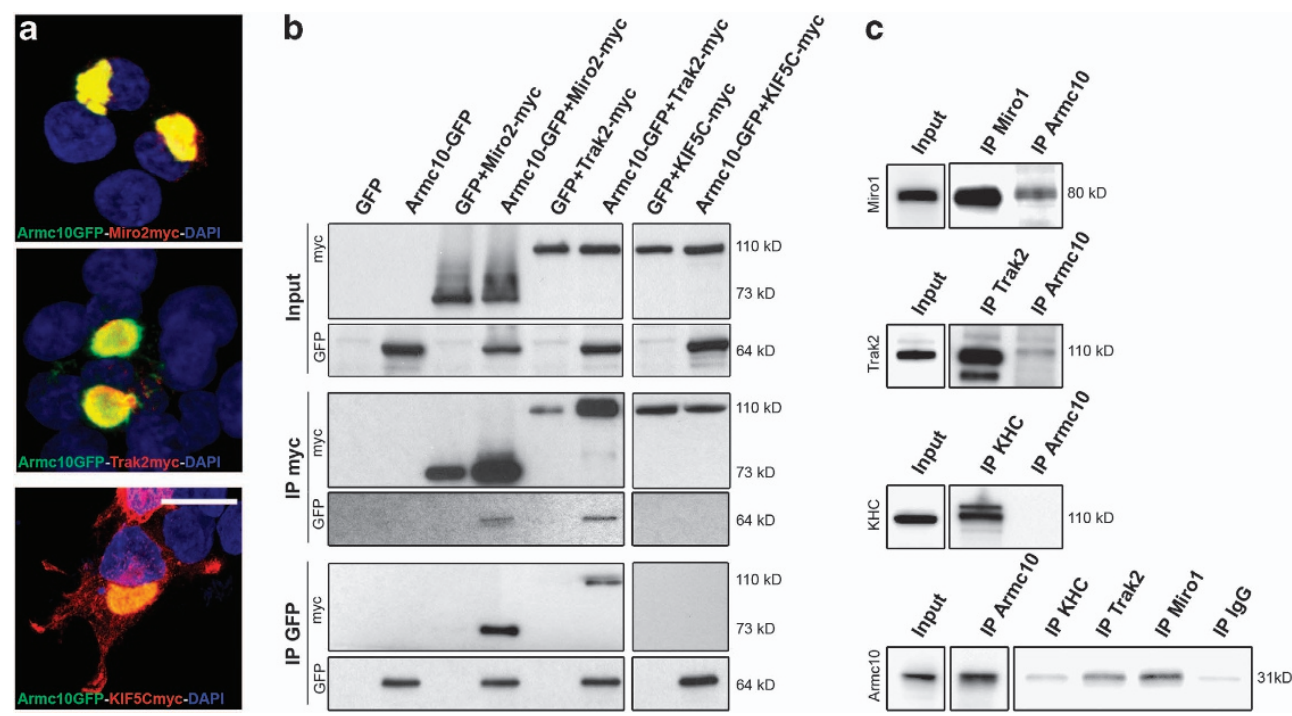

Figure 6 Armc10 colocalises and interacts with Miro1-2 and Trak2 but not with KIF5C proteins. (a) HEK293T cells were transfected with Armc10-GFP and Miro2-myc Trak2-myc or KIF5C-myc and processed for immunocytochemistry. Note the high degree of colocalisation with Miro2 and Trak2, and partial colocalisation with KIF5C. (b) Co-immunoprecipitation experiments in transfected HEK293T cells. Immunoprecipitation with myc antibodies revealed co-association of Miro2-myc and Trak2-myc with Armc10-GFP protein. Reverse immunoprecipitation with GFP antibodies revealed interaction with Miro2-myc and Trak2-myc proteins. No co-association was observed with KIF5C-myc. (c) Immunoprecipitation experiments in adult brain lysates. Immunoprecipitation with Armc10 antibodies reveals co-association with Miro1 and Trak2 but not with KHC (upper three panels). Reverse immunoprecipitation shows interaction of Miro1 and Trak2 with Armc10 (bottom panel). Scale bar: $10 \mu \mathrm{m}$
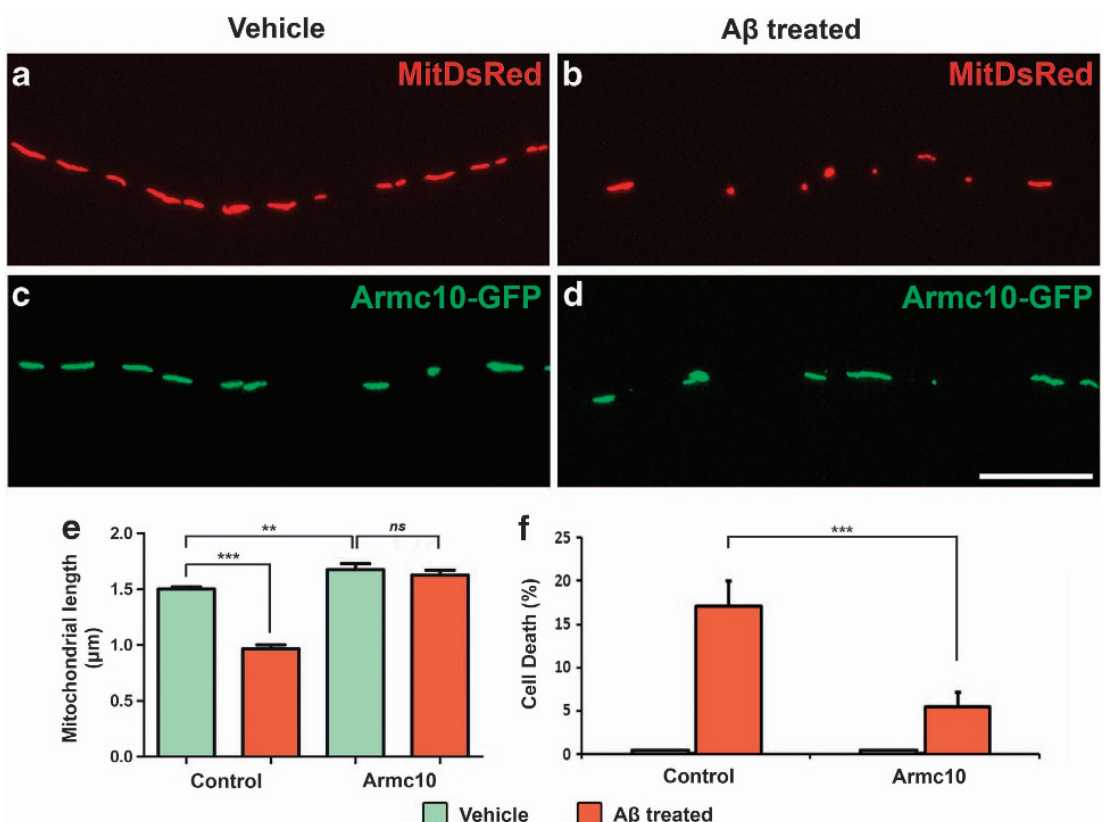

Figure 7 Armc10/SHV protein protects against A $\beta$-induced neuronal death and mitochondrial fragmentation. (a-d) Representative images from cultured hippocampal neurons (axons) transfected with either MitDsRed ( $\mathbf{a}$ and $\mathbf{b}$ ) or Armc10-GFP ( $\mathbf{c}$ and $\mathbf{d}$ ), under control conditions or after A $\beta$ treatment. Note that A $\beta$-induced mitochondrial fragmentation is abolished after overexpression of Armc10. (e) Histogram showing average mitochondrial lengths under the experimental conditions used. (f) Histogram showing the percentage of dead cells (Propidium iodide and Hoechst double-staining) in cultured hippocampal neurons in the same conditions as above and treated with $\mathrm{A} \beta$. Note the reduction in cell death after Armc10 overexpression. Data represent mean \pm S.E.M. $\left({ }^{\star} P<0.05,{ }^{\star \star} P<0.01,{ }^{* \star *} P<0.001\right.$, two-way ANOVA and Bonferroni's post hoc test). Scale bar: $10 \mu \mathrm{m}$

which is present in all the vertebrates surveyed. ${ }^{11}$ In a previous study, we demonstrated that one of the members of the cluster, the Armcx 3 gene, is involved in the regulation of mitochondrial dynamics, aggregation and trafficking in neurons. Here, we investigated whether the Armc10 ancestor gene, from which the entire gene cluster has evolved, is also involved in such mitochondrial regulation. Our data show that the Armc10 protein preferentially localises to nuclei and mitochondria, regulating mitochondrial dynamics and trafficking in neurons through interaction with the KIF5/Miro/Trak2 complex. 
Our analysis of the genomic locus of Armc10 shows that deep synteny is conserved in all tetrapods analysed, with the partial exception of human, which only shares three genes upstream of the Armc10 locus. Our data suggest that a translocation may have occurred in the ancestor of the subfamily Homininae, since gorilla, chimpanzee and human are the only sequenced primates that do not have the gene distribution seen in the remaining tetrapods. In contrast, zebrafish synteny around Armc10 is greatly altered in comparison with other vertebrates, most probably due to gene duplication degeneration after the extra round of genome duplications at the base of the teleost lineage. In addition, we identified three tentative enhancer sequences by combining VISTA and histone mark analyses and searches. The identification of conserved non-coding regions, coupled with determination of histone marks, has been shown to be useful tools in the search for regulatory regions, ${ }^{20,21}$ suggesting possible roles in the regulation of surrounding genes. In the Armc10 genome context, we identified a putative enhancer in the intronic regions of the Armc10 gene, and two in the bystander genes FBXL13 and NAPEPLD. These must be unique for the Armc10 gene, as the ArmcX cluster on the $\mathrm{X}$ chromosome was originated by retrotransposition and further tandem gene duplication and thus does not contain any of the above regions.

In addition to mitochondrial regulation, several members of the Armc10/Armcx gene family bear nuclear localisation signals. ${ }^{11}$ Moreover, it has been reported that Armcx3 interacts with the transcription factor Sox10, thus regulating its transcriptional activity, ${ }^{22}$ and that the Armc10-B isoform controls transcription through interaction with p53. ${ }^{23}$ Furthermore, Armc10/Armcx genes display up to six armadillo domains arrayed in a DUF634 domain. ${ }^{11}$ This trait may indicate the participation of these domains in the Wnt/ $\beta$-Catenin pathway. ${ }^{24,25}$ Thus, although the role of Armc10/Armcx genes in the nucleus is far from being understood, it is probable that this gene cluster encodes a new protein family that may potentially act as a shuttle between the mitochondrial and nuclear compartments.

Our data provide evidence that Armc10 interacts with the Miro 1/2 and Trak2 proteins, two crucial components of the mitochondrial trafficking complex in neurons. ${ }^{26,27}$ These results suggest a model in which the Armc10 protein, through interaction with the Kinesin/Miro/Trak2 complex, favours the formation of this complex, thereby allowing the transport of individual mitochondria. This notion is consistent with the present knockdown experiments, which indicate that downregulation of endogenous Armc10 protein markedly decreases the number of motile mitochondria. The phenotype produced by Armc10 overexpression (also causing mitochondrial arrest) may be due to a dominant-negative effect of Armc10, thus recruiting and/or deregulating several members of the trafficking complex. This effect has also been described for other mitochondria-targeted proteins, including Armcx3 and TDP43. ${ }^{11,28}$ These observations thus suggest that appropriate levels of these proteins are required for normal trafficking. However, we cannot exclude that Armc10 overexpression interferes with mitochondrial trafficking by altering additional unknown mechanisms.
Together with our previous findings, ${ }^{11}$ the present study underscores that the Armc10/Armcx gene family encodes for proteins with evolutionarily conserved functions in the regulation of mitochondrial dynamics and transport. In addition, we found that Armc10 overexpression induces mitochondrial aggregation in HEK293T cells, but not in the hippocampal neurons. This finding could be explained by differential levels of Armc10 expression in the two cell types or by different levels of expression of Armc10-interacting proteins regulating aggregation. Of note, our previous study showed that overexpression of the Armcx 3 gene causes mitochondrial aggregation in both neurons and HEK293 cells. ${ }^{11}$ It is interesting to note that mitochondrial aggregation is believed to be closely related to mitochondrial recycling and mitophagia, ${ }^{29,30}$ thereby suggesting a differential role of several Armc10/ Armcx members in this process.

In addition, we show here again that the Armc10 gene may have unique features in the functional regulation of mitochondrial dynamics. For instance, whereas dysregulation of Armc10 protein levels (both overexpression and downregulation) causes marked mitochondrial arrest, we did not find any evidence for the involvement of the Armc10 protein in the regulation of the velocity of the few mitochondria that remained motile under the two experimental conditions. In contrast, the Armcx3 gene is involved in mitochondrial transport velocity. ${ }^{11}$ This suggests that in contrast to Armcx3, the Armc10 protein has a milder effect, acting as a positive regulator of the KIF5/Miro/Trak2 trafficking complex; however, it does not affect the ATPase activity of kinesins. Taken together, the present data suggest both overlapping and differential mechanisms and roles in the regulation of mitochondrial dynamics and transport by Armc10/Armcx family members.

Finally, we found that Armc10 overexpression causes a slight increment in mitochondrial size under control conditions. This increase may reflect a potential role of Armc10 in mitochondrial fusion/fission. It is interesting to note that both processes, trafficking and fission/fusion, are closely related and are often mediated by the same proteins. ${ }^{3,31}$ Interestingly, Armc10 overexpression prevents mitochondrial fragmentation and neuronal death produced by $\mathrm{A} \beta$ oligomers. Armc 10 mitochondrial fragmentation and fission induced by $\mathrm{A} \beta$ is considered to be a critical event in $\mathrm{A} \beta$-induced neurotoxicity, which normally precedes neuronal death. ${ }^{32}$ Our results thus support a protective role of Armc10 in A $\beta$-induced toxicity. In this context, it is also interesting to note that several members of the Armc10/Armcx gene family are included in the 'core proteome' associated with Parkinson disease and that at least the Armcx3 protein is a substrate of the Parkin/Pink1 ubiquitin/ligase complex involved in the pathogenesis of this disease. ${ }^{33,34}$ Taken together, these findings suggest that regulation of mitochondrial dynamics through the Armc10/ Armcx gene family may be involved in the pathogenesis of neurodegenerative processes.

In conclusion, here we report that the Armc10 gene, the phylogenetic ancestor of the Armcx gene cluster, has a prominent role in the regulation of mitochondrial dynamics and trafficking in neurons. This regulation is probably mediated by its interaction at the protein level with the $\mathrm{KHC} /$ Miro/Trak2 trafficking complex. It is interesting to note that the 
evolutionary appearance of the Armc10 gene in basal chordates is coincidental with the genomic duplication of mitochondrial dynamics-related genes, including mitofusins and the Miro GTPases. ${ }^{35}$ This observation may suggest a coordinated increase in the complexity of the molecular machinery that regulates this process in vertebrates and, specifically, in the most evolved mammals.

Taken together with our previous findings, ${ }^{11}$ our data highlight that the function of the Armc10 ancestor gene in mitochondrial trafficking has been conserved during the evolution and development of the Armcx genomic cluster, although some particular functions may have been acquired during evolution (for example, mitochondrial aggregation). As the Armcx gene cluster arose suddenly during the early evolution of the most evolved mammals, the eutherians, our data suggest an increased complexity in the molecular mechanisms regulating mitochondrial dynamics and trafficking, specifically in the nervous system of higher vertebrates. Finally, and given the protection that the Armc10 exerts against $\mathrm{A} \beta$-induced cell death and mitochondrial fragmentation, our data suggest that the novel Armc10/Armcx gene family of mitochondrial proteins described here may contribute to the pathophysiology of neurological diseases, which often bear mutations or functional dysregulation of proteins controlling mitochondrial dynamics in neurons. ${ }^{2,8}$

\section{Materials and Methods}

All procedures were performed in accordance with the guidelines approved by the Spanish Ministry of Science and Technology and following the European Community Council Directive 86/609 EEC.

Bioinformatics. Sequences for the genomic locus of Armc10 were downloaded from the UCSC Genome Browser (assemblies were: GRCh37 for human; GRCm38 for mouse; loxAfr3 for elephant; monDom5 for opossum; ornAna1 for platypus; galGal4 for chicken; and xenTro3 for Xenopus tropicalis). VISTA $^{36}$ annotations for the human sequence were downloaded from the GRCh37 assembly on the Ensembl Genome Browser (ensembl.org/index.html). ENCODE Project data were accessed via genome.ucsc.edu for H3K4me1 and H3K4me3 marks on all available cell lines. Domain structure was determined by NCBI, Prosite, PPSearch, InterProScan and Wolfsort software. RNA-seq data (available in the Sequence Read Archive (SRA)) was used to quantify Armc10 expression in different tissues, according to the previosuly defined cRPKM expression values. ${ }^{37}$ S.D.s were calculated for tissues with several available expression values.

Plasmid vectors. The full sequence of Armc10 fully fused to myc epitope and shRNAi sequences for this protein were obtained from Origene (MR204310 and TG5187). For the generation of Armc10-GFP expression vectors, the Armc10 sequence was subcloned into the pEGFP-N3 vector (Clontech, Mountain View, CA, USA) and the construct was sequenced with BigDye-Terminator v3.1 (Applied Biosystems, Carlsbad, CA, USA). The sequence of the selected shRNAi-Armc10 was $5^{\prime}$-TCATGATGTGGATGTGAAAGAGAAAGCTTTCAAGAGAAGCTTTCTCTT TCACATCCACATCATGATTTTTTTG-3'; and the scrambled shRNAi-control was 5'-TGACCACCCTGACCTACGGCGTGCAGTGCTCAAGAGGCACTGCACGCC GTAGGTCAGGGTGGTCATTTTTTG-3'. The expression vectors Miro2-myc, KIF5C-myc and Trak2-myc were as previously described. ${ }^{26,38}$ Mitochondrialtargeted DsRed (MitDsRed) was a gift from Antonio Zorzano (IRB Barcelona).

Animals. OF1 embryos and postnatal mice (Iffra Credo, Lyon, France) were used. The mating day was considered embryonic day 0 (EO) and the day of birth, postnatal day 0 (P0). The following developmental stages were studied for histology: E16, P5 and Adult. Animals were anaesthetised with $4 \%$ halothane before killing.

Histology. Embryos and postnatal mice from E16, P5 and Adult stages were fixed in $4 \%$ paraformaldehyde. Brains were cryoprotected, frozen and cut into $50-\mu$ m-thick coronal sections. Immunohistochemistry was performed in freefloating sections. Rabbit anti-Armc10 (1:250, Sigma-Aldrich, Madrid, Spain) was used as primary antibody and immunodetected with a rabbit secondary biotinylated antibody (Vector Laboratories Inc., Burlingame, CA, USA). After streptavidin-peroxidase reaction (Amersham, Piscataway, NJ, USA), sections were developed with $\mathrm{DAB}$, dehydrated and coverslipped.

Cell culture and transfection. HEK293T cells were used for all the experiments. Cells were cultured in DMEM medium supplemented with $10 \%$ FBS, $2 \mathrm{mM}$ glutamine, $120 \mu \mathrm{g} / \mathrm{ml}$ penicillin and $200 \mu \mathrm{g} / \mathrm{ml}$ streptomycin. Upon confluence, cells were trypsinised $(0.25 \% \mathrm{w} / \mathrm{v})$ and plated at the desired density. After 2 days, cells were transfected using Fugene6 (Roche Diagnostics, West Sussex, UK), following the manufacturer's instructions and using a 1:1 DNA ratio when two constructs were transfected. For immunoprecipitation assays, an empty vector was used to balance transfected DNA when required.

E16 mouse brains were dissected in PBS containing $0.6 \%$ glucose and the hippocampi were dissected out. After trypsin (Invitrogen, Carlsbad, CA, USA) and DNAse treatment (Roche Diagnostics), tissue pieces were dissociated and cells were seeded onto $0.5 \mathrm{mg} / \mathrm{ml}$ poly-L-lysine (Sigma-Aldrich)-coated coverslips (for immunocytochemistry) or 35-mm Fluorodish plates (World Precision Instruments Inc) for live-imaging in neurobasal medium (Gibco, Grand Island, NY, USA) containing $2 \mathrm{mM}$ glutamax, $120 \mu \mathrm{g} / \mathrm{ml}$ penicillin, $200 \mu \mathrm{g} / \mathrm{ml}$ streptomycin and B27 supplement (Invitrogen). Cells were maintained at $37^{\circ} \mathrm{C}$ in the presence of $5 \% \mathrm{CO}_{2}$, and were cultured for between 5-6 days. Neuron transfection was carried out at four DIV using Lipofectamine 2000 (Invitrogen), following the manufacturer's instructions and using a 1:3 DNA ratio when transfection of two constructs was required. Cells were processed $24-48 \mathrm{~h}$ after transfection.

For mitochondrial fragmentation analysis, hippocampal neurons were treated for $20 \mathrm{~h}$ before fixation with oligomeric $A \beta$ or vehicle. The $A \beta 1-42$ peptide was purchased from Bachem (Bubendorf, Switzerland). Soluble oligomers of $A \beta 1-42$ were prepared as described previously. ${ }^{39-41}$ Briefly, $60 \mu$ l of $1 \mathrm{mM} \mathrm{A} \beta 1-42$ in HFIP (1,1,1,3,3,3-Hexafluor-2-propanol) was lyophilised and stored at $-80^{\circ} \mathrm{C}$. A working solution of $100 \mu \mathrm{M} \mathrm{A} \beta 1-42$ was prepared by dissolving the lyophilised A $\beta 1-42$ in $12 \mu$ I DMSO plus $600 \mu$ I DMEM-F12 (phenoland glutamine-free), and directly added to the culture medium to a final concentration of $2 \mu \mathrm{M}$. Control cultures were treated with the same volume of DMSO/DMEM-F12 vehicle.

Immunocytochemistry. HEK293T cells or neurons were fixed in $4 \%$ paraformaldehyde. After fixation, cells were permeabilised with Triton X-100 in PBS and blocked with blocking buffer (10\% fetal bovine serum (Roche Diagnostics), $0.2 \mathrm{M}$ glycine, $0.1 \%$ Triton $\mathrm{X}-100$ and $0.05 \%$ deoxycholic acid in PBS- $2 \%$ gelatin) for $1 \mathrm{~h}$ at room temperature. To label the cells, the following antibodies or dyes were used: rabbit anti-Armc10 (1:250, Sigma-Aldrich), rabbit anti-GFP (1:500, Invitrogen) and mouse anti-myc (1:500, Santa Cruz Biotechnology, Heidelberg, Germany) in blocking buffer for $2 \mathrm{~h}$ and with the corresponding secondary antibodies labelled with fluorochromes (Alexafluor 546 or 488, Invitrogen). Nuclei were stained with the specific dye Hoechst-33342. All samples were mounted on Mowiol.

Imaging and quantification of axonal transport of mitochondria. Hippocampal neurons were seeded onto Poly-L-lysine-coated Fluorodish plates (World Precision Instruments Inc), transfected with Armc10-GFP and MitDsRed and filmed 24-48 h later using a Leica TCS SP2 confocal microscope (Leica Microsystems) equipped with a $\times 63$ immersion oil objective. Axonal mitochondria were registered with an additional digital zoom of $\times 1.7$. Time-lapse series of image stacks composed of five images $(512 \times 512 \mathrm{px})$ were taken every $6 \mathrm{~s}$ for $15 \mathrm{~min}$. All 151 stacks obtained were processed with the Leica Confocal Software. Further image processing, analysis and video compilation (10 frames per second) and edition was carried out using the ImageJ software (version 1.43K, NIH, Bethesda, MD, USA). Kymographs were generated with MetaMorph Software (Molecular Devices - MDS Analytical Technologies, Sunnyvale, CA, USA). In overexpression experiments, 17 axons were registered and analysed for each condition, while in silencing experiments 12 axons per group were recorded. In all cases, a mitochondrion was considered motile when it moved more than $0.5 \mu \mathrm{m}$ during $1 \mathrm{~min}$ of recording. Distances and speeds of retrograde and anterograde transport were measured separately from the corresponding kymographs, as previously described, ${ }^{42}$ and no trackingplugging was used. 
Imaging and quantification of mitochondrial length. Visualisation and capture were performed using a $\times 100 / 1.4$ oil immersion objective on a Nikon Eclipse E1000 microscope with an attached Olympus DP72 digital camera. Images were processed with the NIH ImageJ 'Analyse Particles' module measuring the Feret's diameter of thresholded mitochondria. The length and number of mitochondria were measured in a $50-\mu \mathrm{m}$ axonal section per neuron. Results are expressed as average mitochondrial length per axonal section.

Isolation of brain mitochondria and cell fractionation. The brains of Adult C57BL6/J mice were dissected, and mitochondria were purified as described. ${ }^{43}$ Briefly, brains were extracted in ice-cold isolation buffer (sucrose $250 \mathrm{mM}$, Tris $10 \mathrm{mM}$, EDTA $1 \mathrm{mM}, \mathrm{NaF} 2 \mathrm{mM}, \mathrm{pH} 7.6$ ) and homogenized with a Teflon potter. Homogenates were centrifuged at $1500 \mathrm{~g}$ for $5 \mathrm{~min}\left(4^{\circ} \mathrm{C}\right)$. The supernatant was then centrifuged at $12500 \mathrm{~g}\left(4^{\circ} \mathrm{C}\right)$. The pellet was collected, and both centrifugations were repeated. To purify mitochondria, the final pellet was resuspended in $400 \mu \mathrm{l}$ of isolation buffer, layered on top of a discontinuous Ficoll gradient (10 and 7\% fractions), and centrifuged at $100000 \mathrm{~g}$ for $1 \mathrm{~h}\left(4^{\circ} \mathrm{C}\right)$. The following antibodies were used as purification controls: anti-SDHA (1:20000, Abcam, Cambridge, UK) and anti-TOM20 (1:1000, Santa Cruz Biotechnology) as mitochondrial markers; anti-LDHa (1:500, Santa Cruz Biotechnology) as cytosolic marker; anti-tubulin (1:1000, Sigma-Aldrich) as cytoskelton marker; and anti-synaptophysin (1:20000, Millipore, Schwalbach, Germany) as synaptosome marker.

Propidium iodide staining. To quantify $A \beta$-induced cell death, hippocampal neurons were seeded in 24-well plates and transfected with Armc10-GFP or GFP. Cultures were treated with A $\beta 1-42$ as described above. After $24 \mathrm{~h}$, cells were incubated for $1 \mathrm{~h}$ with propidium iodide $(1.5 \mu \mathrm{m} / \mu \mathrm{l})$ and counterstained with Hoechst $33342(1 \mu \mathrm{g} / \mu \mathrm{l})$. Cells were than washed three times with PBS and fixed for $20 \mathrm{~min}$ with $0.1 \mathrm{M} \mathrm{PB}$ containing $4 \%$ of paraformaldehyde. Fluorescent micrographs were randomly taken from coverslips for each condition. The number of dying neurons ( $\mathrm{PI}$ and Hoechst double-labelled pyknotic nuclei) versus the total number of cells (Hoechst-labelled nuclei) per field, were counted. Data represent eight fields per condition and experiment (from two independent experiments).

Protein cell extracts, WB and immunoprecipitation. HEK293T cells were obtained and lysed in Laemmli Buffer (LB) at $98^{\circ} \mathrm{C}$ for $5 \mathrm{~min}$. Twenty micrograms of protein for each sample were loaded and run in polyacrylamide gels at $100 \mathrm{~V}$. Transfer to nitrocellulose membranes was performed in $120 \mathrm{mM}$ glycine, $125 \mathrm{mM}$ Tris, $0.1 \%$ SDS and $20 \%$ methanol at $35 \mathrm{~V}$ o.n. Membranes were then blocked in TBS containing 5\% non-fat dry milk and incubated with the primary antibodies anti-Armc10 (1:1000, Sigma-Aldrich), anti-GFP (1:1000, Invitrogen) and anti-myc (1:2000, Santa Cruz Biotechnology). Anti-actin was used as a loading control (1:1000, Chemicon, Temecula, CA, USA). Secondary antibodies coupled to HRP were used in a 1:2500 dilution in TBS containing $5 \%$ non-fat dry milk. Labelling was visualised with ECL plus (Amersham Pharmacia Biotech).

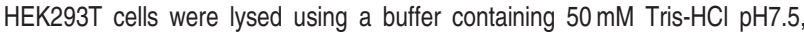
$150 \mathrm{mM} \mathrm{NaCl}, 1.5 \mathrm{mM} \mathrm{MgCl}, 5 \mathrm{mM}$ EDTA, $1 \%$ Triton-100, 10\% glycerol, protease inhibitor cocktail (Roche Diagnostics $\mathrm{Gmbh}$ ), $1 \mathrm{mM} \mathrm{NaF}, 0.5 \mathrm{M}$ sodium pyrophosphate and $200 \mathrm{mM}$ orthovanadate. Five hundred micrograms of total protein per sample were used for the immunoprecipitation assays. Homogenates were incubated overnight with anti-GFP $(1: 500$, Invitrogen) and anti-myc $(1: 500$, Santa Cruz Biotechnology) at $4^{\circ} \mathrm{C}$. All the assays were performed for $2 \mathrm{~h}$ at $4^{\circ} \mathrm{C}$ using protein G-Sepharose beads (Sigma-Aldrich). After incubation with protein G-Sepharose, samples were washed five times in washing buffer $(10 \mathrm{mM}$ Tris$\mathrm{HCl} \mathrm{pH8}, 500 \mathrm{mM} \mathrm{NaCl}, 1 \mathrm{mM}$ EDTA, $1 \mathrm{mM}$ EGTA, $1 \%$ Triton X-100 and $0.5 \% \mathrm{NP}$ 40). LB was added to the beads, and they were then boiled at $98{ }^{\circ} \mathrm{C}$ for $5 \mathrm{~min}$. Proteins were then analysed using SDS-PAGE and WB.

Adult brain extracts were obtained by homogenisation in lysis buffer $(50 \mathrm{mM}$ Tris- $\mathrm{HCl}, 150 \mathrm{mM} \mathrm{NaCl}, 5 \mathrm{mM}$ EGTA, 10\% glycerol, 1\% Triton X-100, protease inhibitor cocktail (Roche Diagnostics Gmbh), $1 \mathrm{mM}$ PMSF and $200 \mathrm{mM}$ orthovanadate). Ten milligrams were incubated with $1 \mu \mathrm{g}$ of antibodies anti-KHC (Chemicon), anti-Miro1 (Sigma), anti-Trak2 (Sigma) and anti-Armc10 (Proteintech, Chicago, IL, USA). Immunoprecipitation was performed using Protein G Magnetic Beads (Invitrogen) following the manufacturer's instructions. After this procedure, the beads were washed three times with PBS, eluted in SDS sample buffer for $5 \mathrm{~min}$ at $85^{\circ} \mathrm{C}$ and subjected to WB with the corresponding antibodies.
Statistical analysis. Statistical analyses of differences were conducted using one-way ANOVA with Bonferroni post hoc comparisons to compare three or more groups or with Student's $t$-test when required. Statistical significance was fixed at $P<0.05 ;{ }^{*} P<0.05,{ }^{*} P<0.01$ and ${ }^{* \star *} P<0.001$.

\section{Conflict of Interest}

The authors declare no conflict of interest.

Acknowledgements. This project was supported by grants BFU2008-3980 and SAF2011-13232-E (Acciones Complementarias) (MINECO, Spain) to ES, by grant BFU2010-21507 to FU, by grant SAF2011-23550 to RT, by grant 'BFU201123921' and the ICREA Academia Prize (Generalitat de Catalunya) to JGF, and by a grant from the CIBERNED. SM and EN hold FPI fellowships. We thank Ashraf Muhaisen for technical help and Tanya Yates for editorial assistance.

1. Nicholls DG, Budd SL. Mitochondria and neuronal survival. Physiol Rev 2000; 80: 315-360

2. Han XJ, Tomizawa K, Fujimura A, Ohmori I, Nishiki T, Matsushita M et al. Regulation of mitochondrial dynamics and neurodegenerative diseases. Acta Med Okayama 2011; 65: $1-10$

3. Misko A, Jiang S, Wegorzewska I, Milbrandt J, Baloh RH. Mitofusin 2 is necessary for transport of axonal mitochondria and interacts with the Miro/Milton complex. J Neurosci 2010; 30: 4232-4240

4. Hoppins S, Lackner L, Nunnari J. The machines that divide and fuse mitochondria. Annu Rev Biochem 2007; 76: 751-780.

5. Guo X, Macleod GT, Wellington A, Hu F, Panchumarthi S, Schoenfield M et al. The GTPase dMiro is required for axonal transport of mitochondria to Drosophila synapses. Neuron 2005; 47: 379-393.

6. Pilling $A D$, Horiuchi D, Lively $C M$, Saxton WM. Kinesin-1 and Dynein are the primary motors for fast transport of mitochondria in Drosophila motor axons. Mol Biol Cell 2006; 17: 2057-2068.

7. Stowers RS, Megeath LJ, Gorska-Andrzejak J, Meinertzhagen IA, Schwarz TL. Axonal transport of mitochondria to synapses depends on milton, a novel Drosophila protein. Neuron 2002; 36: 1063-1077.

8. Chen $\mathrm{H}$, Chan DC. Mitochondrial dynamics-fusion, fission, movement, and mitophagy-in neurodegenerative diseases. Hum Mol Genet 2009; 18: R169-R176.

9. Schon EA, Przedborski S. Mitochondria: the next (neurode)generation. Neuron 2011; 70 : 1033-1053

10. Perier C, Vila M. Mitochondrial biology and Parkinson's disease. Cold Spring Harb Perspect Med 2012; 2: a009332.

11. Lopez-Domenech G, Serrat R, Mirra S, D'Aniello S, Somorjai I, Abad A et al. The Eutherian Armcx genes regulate mitochondrial trafficking in neurons and interact with Miro and Trak2. Nat Commun 2012; 3: 814

12. Hollenbeck PJ, Saxton WM. The axonal transport of mitochondria. J Cell Sci 2005; 118(Pt 23): $5411-5419$

13. Hollenbeck PJ. The pattern and mechanism of mitochondrial transport in axons. Front Biosci 1996; 1: d91-102.

14. MacAskill AF, Kittler JT. Control of mitochondrial transport and localization in neurons. Trends Cell Biol 2010; 20: 102-112.

15. Heintzman ND, Stuart RK, Hon G, Fu Y, Ching CW, Hawkins RD et al. Distinct and predictive chromatin signatures of transcriptional promoters and enhancers in the human genome. Nat Genet 2007; 39: 311-318.

16. Huang R, Xing Z, Luan Z, Wu T, Wu X, Hu G. A specific splicing variant of SVH, a novel human armadillo repeat protein, is up-regulated in hepatocellular carcinomas. Cancer Res 2003; 63: 3775-3782

17. Rapaport D. Finding the right organelle. Targeting signals in mitochondrial outermembrane proteins. EMBO Rep 2003; 4: 948-952.

18. Manczak M, Reddy PH. Abnormal interaction of VDAC1 with amyloid beta and phosphorylated tau causes mitochondrial dysfunction in Alzheimer's disease. Hum $\mathrm{Mol}$ Genet 2012; 21: 5131-5146.

19. Wang X, Su B, Siedlak SL, Moreira PI, Fujioka H, Wang $Y$ et al. Amyloid-beta overproduction causes abnormal mitochondrial dynamics via differential modulation of mitochondrial fission/fusion proteins. Proc Natl Acad Sci USA 2008; 105: 19318-19323.

20. Roh TY, Wei G, Farrell CM, Zhao K. Genome-wide prediction of conserved and nonconserved enhancers by histone acetylation patterns. Genome Res 2007; 17: 74-81.

21. Heintzman ND, Hon GC, Hawkins RD, Kheradpour P, Stark A, Harp LF et al. Histone modifications at human enhancers reflect global cell-type-specific gene expression. Nature 2009; 459: 108-112.

22. Mou Z, Tapper AR, Gardner PD. The armadillo repeat-containing protein, ARMCX3, physically and functionally interacts with the developmental regulatory factor Sox10. J Biol Chem 2009; 284: 13629-13640.

23. Zhou $X$, Yang G, Huang $R$, Chen $X, H u$ G. SVH-B interacts directly with p53 and suppresses the transcriptional activity of p53. FEBS Lett 2007; 581: 4943-4948. 
24. Coveney D, Ross AJ, Slone JD, Capel B. A microarray analysis of the XX Wnt4 mutant gonad targeted at the identification of genes involved in testis vascular differentiation. Gene Exp Patterns 2008; 8: 529-537.

25. Iseki H, Takeda A, Andoh T, Takahashi N, Kurochkin IV, Yarmishyn A et al. Human Arm protein lost in epithelial cancers, on chromosome X 1 (ALEX1) gene is transcriptionally regulated by CREB and Wnt/beta-catenin signaling. Cancer Sci 2010; 101: 1361-1366.

26. Macaskill AF, Rinholm JE, Twelvetrees AE, Arancibia-Carcamo IL, Muir J, Fransson A et al. Miro1 is a calcium sensor for glutamate receptor-dependent localization of mitochondria at synapses. Neuron 2009; 61: 541-555.

27. Wang $X$, Schwarz TL. The mechanism of $\mathrm{Ca} 2+$-dependent regulation of kinesin-mediated mitochondrial motility. Cell 2009; 136: 163-174.

28. Wang W, Li L, Lin WL, Dickson DW, Petrucelli L, Zhang T et al. The ALS disease-associated mutant TDP-43 impairs mitochondrial dynamics and function in motor neurons. Hum Mol Genet 2013; 22: 4706-4719.

29. Kawajiri S, Saiki S, Sato S, Sato F, Hatano T, Eguchi H et al. PINK1 is recruited to mitochondria with parkin and associates with LC3 in mitophagy. FEBS Lett 2010; 584: 1073-1079.

30. Kaushik S, Cuervo AM. Chaperone-mediated autophagy: a unique way to enter the lysosome world. Trends Cell Biol 2012; 22: 407-417.

31. Baloh RH, Schmidt RE, Pestronk A, Milbrandt J. Altered axonal mitochondrial transport in the pathogenesis of Charcot-Marie-Tooth disease from mitofusin 2 mutations. J Neurosci 2007; 27: 422-430.

32. Zhu X, Perry G, Smith MA, Wang X. Abnormal mitochondrial dynamics in the pathogenesis of Alzheimer's disease. J Alzheimers Dis 2013; 33(Suppl 1): S253-S262.

33. Moran LB, Graeber MB. Towards a pathway definition of Parkinson's disease: a complex disorder with links to cancer, diabetes and inflammation. Neurogenetics 2008; 9: 1-13.

34. Sarraf SA, Raman M, Guarani-Pereira V, Sowa ME, Huttlin EL, Gygi SP et al. Landscape of the PARKIN-dependent ubiquitylome in response to mitochondrial depolarization. Nature 2013; 496: 372-376.

35. Vlahou G, Elias M, von Kleist-Retzow JC, Wiesner RJ, Rivero F. The Ras related GTPase Miro is not required for mitochondrial transport in Dictyostelium discoideum. Eur J Cell Biol 2011; 90: 342-355.

36. Frazer KA, Pachter L, Poliakov A, Rubin EM, Dubchak I. VISTA: computational tools for comparative genomics. Nucleic Acids Res 2004; 32: W273-W279.
37. Labbe RM, Irimia M, Currie KW, Lin A, Zhu SJ, Brown DD et al. A comparative transcriptomic analysis reveals conserved features of stem cell pluripotency in planarians and mammals. Stem Cells 2012; 30: 1734-1745.

38. MacAskill AF, Brickley K, Stephenson FA, Kittler JT. GTPase dependent recruitment of Grif-1 by Miro1 regulates mitochondrial trafficking in hippocampal neurons. Mol Cell Neurosci 2009; 40: 301-312.

39. Lambert MP, Barlow AK, Chromy BA, Edwards C, Freed R, Liosatos M et al. Diffusible, nonfibrillar ligands derived from Abeta1-42 are potent central nervous system neurotoxins. Proc Natl Acad Sci USA 1998; 95: 6448-6453.

40. Dahlgren KN, Manelli AM, Stine WB Jr, Baker LK, Krafft GA, LaDu MJ. Oligomeric and fibrillar species of amyloid-beta peptides differentially affect neuronal viability. J Biol Chem 2002; 277: 32046-32053.

41. Klein WL. Abeta toxicity in Alzheimer's disease: globular oligomers (ADDLs) as new vaccine and drug targets. Neurochem Int 2002; 41: 345-352.

42. De Vos KJ, Sheetz MP. Visualization and quantification of mitochondrial dynamics in living animal cells. Methods Cell Biol 2007; 80: 627-682.

43. Benard G, Faustin B, Passerieux E, Galinier A, Rocher C, Bellance N et al. Physiological diversity of mitochondrial oxidative phosphorylation. Am J Physiol Cell Physiol 2006; 291: C1172-C1182.

(i) $(9)($ Cell Death and Disease is an open-access journal published by Nature Publishing Group. This work is licensed under a Creative Commons Attribution-NonCommercialNoDerivs 3.0 Unported License. The images or other third party material in this article are included in the article's Creative Commons license, unless indicated otherwise in the credit line; if the material is not included under the Creative Commons license, users will need to obtain permission from the license holder to reproduce the material. To view a copy of this license, visit http://creativecommons.org/licenses/ by-nc-nd/3.0/

Supplementary Information accompanies this paper on Cell Death and Disease website (http://www.nature.com/cddis) 